\title{
LA IMPORTANCIA DE LA EDUCACIÓN AMBIENTAL SUSTENTABLE PARA LA PROTECCIÓN DE LA BIODIVERSIDAD EN UNA FACULTAD DE SUDÁFRICA
}

\author{
THE IMPORTANCE OF SUSTAINABLE ENVIRONMENTAL EDUCATION FOR \\ THE PROTECTION OF BIODIVERSITY IN A FACULTY OF SOUTH AFRICA
}

\author{
A IMPORTÂNCIA DA SUSTAINABLE EDUCAÇÃO AMBIENTAL PARA A \\ PROTECÇÃO DA BIODIVERSIDADE EM UMA FACULDADE DE ÁFRICA DO \\ SUL
}

Ramón Bedolla Solano ${ }^{1}$

\begin{abstract}
RESUMO
Referindo-se à crise ambiental que acontece, ele está falando sobre a perda de biodiversidade, entre outros. É uma preocupação. Na educação, é necessário incorporar a Educação Ambiental Sustentável (EAS), em razão de dirigir habilidades para o meio. Este artigo teve como objetivo identificar a importância dada à Educação Ambiental Sustentável (EAS) para o cuidado da biodiversidade em todo o currículo. O estudo foi conduzido na Faculdade de Educação pertencente a Universidade Metropolitana Nelson Mandela na África do Sul. Tendo em vista o objectivo declarado, baseou-se no questionamento professores e alunos sobre como eles percebem as questões ambientais, biodiversidade e incorporando (EAS) no currículo para determinar a sua importância. Os resultados mostraram que os problemas ambientais e da biodiversidade de forma ambígua perceber, no entanto, o (EAS) no currículo não listados.
\end{abstract}

PALAVRAS-CHAVE: Sustentável Educação Ambiental. Biodiversidade. Curriculum. Professor.

Estudante.

\begin{abstract}
Referring to the environmental crisis that happens, is talking about the loss of biodiversity, among others. It is a matter of concern. In the educational field, it is necessary to incorporate Sustainable Environmental Education (SEE) for the reason of guiding competencies in favor of the environment. The objective of this article was to identify the importance given to Sustainable Environmental Education (SEE) for the care of biodiversity through the curriculum. The study was conducted at the Faculty of Education belonging to Nelson Mandela Metropolitan University in South Africa. In response to the objective, it was based on questioning teachers and students about how they perceive the environmental problem, biodiversity and the incorporation of (SEE) in the curriculum to determine its importance. The results obtained showed that the environmental problem and biodiversity are perceived ambiguously, however, (SEE) in the curriculum does not figure.
\end{abstract}

KEYWORDS: Sustainable Environmental Education. Biodiversity. Curriculum. Teacher. Student.

\section{RESUMEN}

Referirse a la crisis ambiental que acontece, es hablar de la perdida de la biodiversidad, entre otros. Es un

\footnotetext{
${ }^{1}$ Universidad Autónoma de Guerrero, México
}

\begin{tabular}{l|l} 
Recebido em: 14/06/2018 & Aceito em: 04/09/2018
\end{tabular}




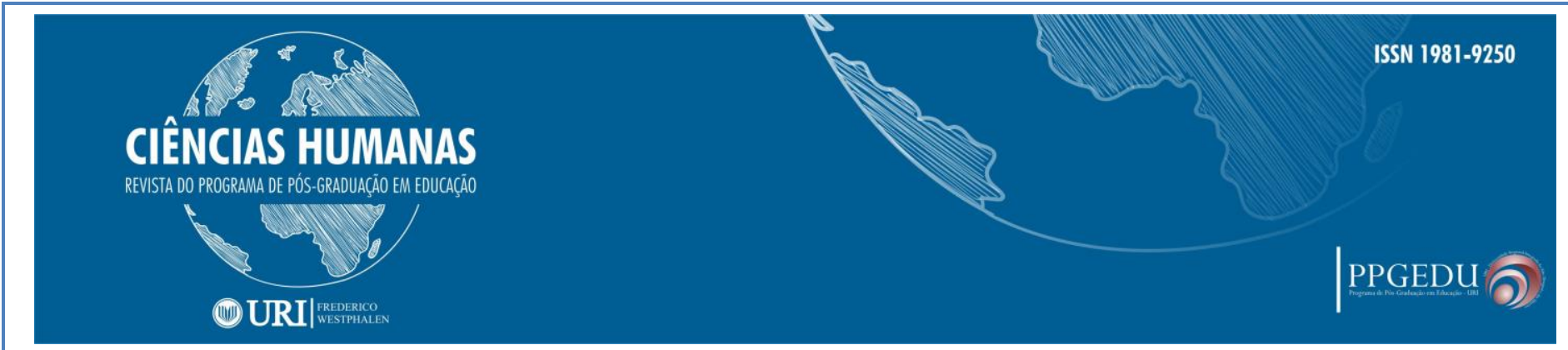

tema preocupante. En el ámbito educativo, es necesario incorporar la Educación Ambiental Sustentable (EAS) por la razón de orientar competencias en pro del medio. El presente artículo tuvo como objetivo, identificar la importancia que se da a la Educación Ambiental Sustentable (EAS) para el cuidado de la biodiversidad a través del currículo. El estudio fue realizado en la Facultad de Educación perteneciente a la Universidad Metropolitana Nelson Mandela en Sudáfrica. En atención al objetivo planteado, se basó en cuestionar docentes y estudiantes sobre la manera en que perciben la problemática ambiental, la biodiversidad y la incorporación de (EAS) en el currículo para determinar su importancia. Los resultados obtenidos mostraron que la problemática ambiental y la biodiversidad se perciben ambiguamente, sin embargo, la (EAS) en el currículo no figura.

PALABRAS CLAVE: Educación Ambiental Sustentable. Biodiversidad. Currículo. Docente.

Estudiante.

\section{INTRODUCCIÓN}

El mundo enfrenta una crisis severa respecto al ambiente, son muchos los problemas que lo aquejan y estos influyen en su deterioro, esto provoca un impacto negativo en la biodiversidad o diversidad biológica, dicho concepto en estas líneas hace alusión a las diversas formas de vida que se desarrollan, como lo son plantas y animales, algunas especies han desaparecido y si no se actúa a tiempo pueden desaparecer muchas más. Los efectos producidos por las actividades humanas son los causantes de las problemáticas que alteran el medio y por ende, los ecosistemas. La falta de cultura y la formación de un sentido crítico para actuar en pro del medio es importante y necesario para que las personas comprendan que debemos de cuidar y preservar los recursos que la naturaleza nos ofrece, por esta razón, resulta importante la incorporación de la Educación Ambiental con enfoque sustentable o del desarrollo en el ámbito educativo o siendo más específicos en los currículos escolares. Las reuniones y cumbres que han tocado la temática "medio ambiente" que han tenido lugar en diferentes partes del mundo como la desarrollada en Suecia en 1972, Rio de Janeiro en 1992 y Johannesburgo en el año 2002 entre otras, han concluido en la importancia de incorporar la Educación Ambiental (enfoque educativo, naturalista o conservador, educar para la naturaleza) y la Educación Ambiental para el Desarrollo Sostenible (enfoque educativo ambientalista, que integra naturaleza, sociedad, economía) en la Educación Formal, como una estrategia para despertar y promover actitudes favorables a favor del medio. La implementación de la Educación Ambiental en la educación formal se ha desarrollado a través de dos enfoques, el conservador, con tintes como su nombre lo indica o mercantilista, comprendido ello como educar para la naturaleza y 


\section{CIÊNCIAS HUMANAS}

REVISTA DO PROGRAMA DE PÓS-GRADUAĞ̈O EM EDUCAĞ̄o

\section{(10)URI|}

confundiéndose con "ecología", en el aspecto pedagógico, apegado a paradigmas positivistas o tradicionales. El ambientalista, con tintes integradores y apegado a las teorías del desarrollo que incorpora los elementos (naturaleza, sociedad, economía), cabe mencionar que puede integrar otros elementos, no obstante, se consideran los más representativos, en el aspecto pedagógico, este se desarrolla a través de métodos constructivistas y actualmente de competencias. Las instituciones educativas de todos los niveles educativas en todo el mundo, de acuerdo a los resolutivos de las reuniones y cumbres realizadas, dan por hecho que la Educación Ambiental debe ser integrada en sus currículos, de tal manera que los individuos promuevan conocimientos significativos respecto a la crisis ambiental que aqueja al contexto y que afecte la diversidad biológica.

Teniendo presente lo estipulado en el párrafo anterior, este estudio, planteó entre sus objetivos determinar la presencia de la Educación Ambiental con enfoque del desarrollo sostenible en el currículo de la Facultad de Educación perteneciente a la Universidad Metropolitana Nelson Mandela en Puerto Elizabeth Sudáfrica, la incorporación de la (EAS) permitiría comprender la importancia que se le da en el plan de estudios de esta institución. Incorporar (EAS), es un proceso amplio, no es una materia en si (si se incorpora con una metodología transversal en el currículo) implica comprender, analizar, actuar y proponer sobre diferentes temas que se relacionan con lo ambiental, en este caso, sería mezclar lo educativo con la biodiversidad, la biodiversidad con lo social, lo económico, etc. La Educación Ambiental de acuerdo a los resolutivos de las reuniones realizadas en diversas partes del mundo, esta, debe ser integrada en todos los niveles educativos escolares y por supuesto que las escuelas de África no deben ser la excepción. En Johannesburgo, Sudáfrica en el 2002, tuvo lugar la Cumbre de la Tierra y en ella, se reforzaron los lineamientos de reuniones que antecedieron para implementar la Educación Ambiental con enfoque del desarrollo y con pedagogías constructivistas. África es un continente con mucha biodiversidad, muchas especies de plantas y animales abundan ahí, por consiguiente, las instituciones educativas deben considerar la (EAS) para protegerla y preservarla. Los resultados obtenidos en la institución donde se realizó esta investigación mostraron que a través 


\section{CIÊNCIAS HUMANAS}

REVISTA DO PROGRAMA DE PÓS-GRADUAC̄̄o EM EDUCACĞ̄o

\section{(1) URI|}

del currículo no se da importancia a la Educación Ambiental para proteger la biodiversidad debido a que en primer lugar el currículo no incorpora dicho elemento, los docentes no desarrollan competencias de (EAS) y los estudiantes no promueven competencias para proteger y preservar la biodiversidad.

Problemáticas relacionadas con la biodiversidad:

Se entiende, por diversidad biológica la variabilidad de organismos vivos de cualquier fuente incluidos, entre otras cosas, los ecosistemas terrestres y marinos. La Ley de Bases Generales del Medio Ambiente de Chile en su artículo $2^{\mathrm{a}}$ Letra a), disposición que hace sinónimos los conceptos de biodiversidad y diversidad biológica. Es necesario ligar este concepto, desde la perspectiva de la protección, con otros elementos tales como la Conservación del Patrimonio Ambiental, la Conservación, el Desarrollo Sustentable y Recursos Naturales, principalmente (Figueroa, 2005: 320-322). La pérdida de la diversidad, como consecuencia de la acción voluntaria del hombre a través de la contaminación, caza de especies que se encuentran en proceso de extinción, entre otras cuestiones, se da por las maniobras y falta de conciencia de algunos seres humanos en su obrar (DefinicionABC).

En el Informe de la Cumbre Mundial sobre el Desarrollo Sostenible en el capítulo I, Anexo Declaración de Johannesburgo sobre el Desarrollo Sostenible, en el punto, los grandes problemas que debemos resolver, se establece en el punto 13 que, "el medio ambiente mundial sigue deteriorándose. Continúa la pérdida de biodiversidad; siguen agotándose las poblaciones de peces; la desertificación avanza cobrándose cada vez más tierras fértiles; ya se hacen evidentes los efectos adversos del cambio del clima; los desastres naturales son más frecuentes y más devastadores, y los países en desarrollo se han vuelto más vulnerables, en tanto que la contaminación del aire, el agua y los mares sigue privando a millones de seres humanos de una vida digna". La biodiversidad del planeta está siendo severamente afectada por las modificaciones, sin precedentes, inducidas por las actividades humanas sobre los ecosistemas, entre las cuales destacan el cambio de usos del suelo, la alteración de los ciclos biogeoquímicos, la destrucción y fragmentación de hábitats, la introducción de especies exóticas y la alteración de las 


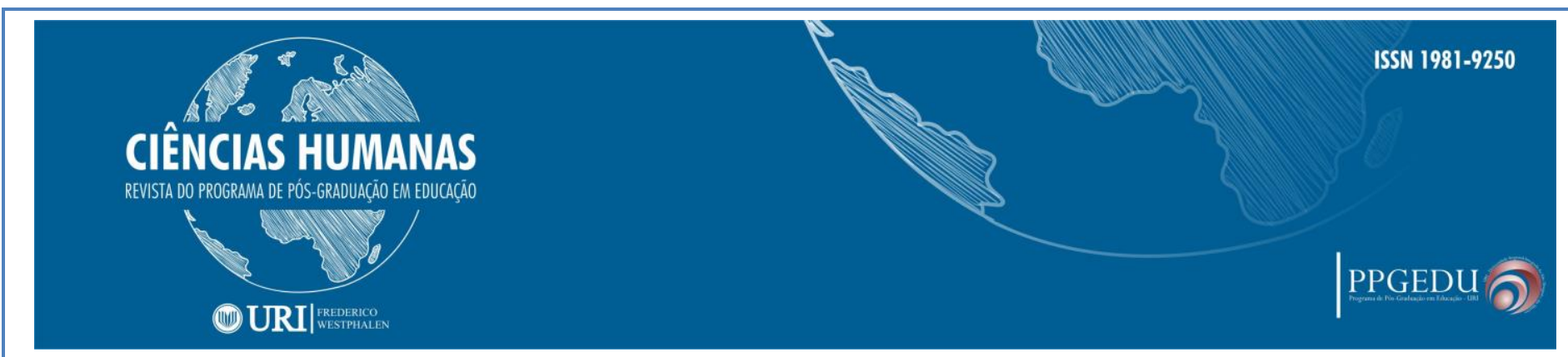

condiciones climáticas (MEA, 2005:Martin et al., 2007a: 1). Por otra parte, aunque no haya sido tan ampliamente reconocido, existen también claras evidencias de que los cambios en la biodiversidad están repercutiendo directa o indirectamente sobre el bienestar humano, ya que comprometen el funcionamiento mismo de los ecosistemas y su capacidad de generar servicios esenciales para la sociedad (Díaz et al., 2006: Martin et al., 2007b: 1). Los problemas son representados por la contaminación, degradación, tráfico de animales, cambio climático, etc. y son atribuidos al ser humano por su escaso nivel cultural que posee. En muchos países alrededor del mundo, los científicos han encontrado muchas evidencias de cambios en el agua, en el crecimiento de las plantas, en donde los animales viven y donde las enfermedades ocurren, claramente ello es debido a cambios que ocurren frecuentemente en nuestro planeta, se le llama cambio climático (Treat, 2006:2). El modelo de desarrollo económico actual ha traído como consecuencia una serie de problemas ambientales como la pérdida de suelos, de recursos hídricos, contaminación de residuos sólidos peligrosos y no peligrosos, contaminación visual, cambio climático, deforestación y pérdida de la biodiversidad, los cuales han afectado gravemente a los ecosistemas del planeta. De no afrontarse este deterioro a corto plazo, se pueden tener dificultades de escasez de los recursos naturales renovables y no renovables (Espejel et al., 2012: 1). La situación de la emergencia planetaria generada por la actividad humana desde mediados del siglo $\mathrm{XX}$, se ha manifestado en problemas ambientales, la pérdida de diversidad biológica y cultural; que tienen como causas evidentes el hiperconsumo, la explotación, y transición demográfica, las inequidades y desequilibrios humanos, la actividad de las organizaciones mafiosas, la actividad especuladora de las empresas transnacionales y la urbanización creciente y desordenada (Mora-Penagos, 2012: Edward et al., 2004: Bedolla et al., 2014: 14-21). Un estudio realizado con estudiantes de la Benemérita Universidad Autónoma de Puebla para describir la representación social de las causas atribuidas a los problemas ambientales, encontró que estas se atribuyen principalmente a causas culturales -inconsciencia, falta de educación e irresponsabilidad-; seguidas de causas económicas -sobreexplotación-; políticas -gobierno corrupto-; tecnológicas fábricas y autos-; sobrepoblación y urbanización; sociales y naturales. Se concluye que 


\section{CIÊNCIAS HUMANAS}

REVISTA DO PROGRAMA DE PÓS-GRADUAĞ̈O EM EDUCAĞ̄o

\section{(1) URI|}

el paso por la universidad influye poco en la representación que tienen los alumnos sobre las causas de los problemas ambientales (Lara et al., 2010: 40).

\section{Desarrollo Sostenible para África}

El desarrollo sostenible ha seguido siendo difícil de alcanzar para muchos países de África. La pobreza sigue siendo un gran obstáculo y la mayoría de los países del continente no han podido aprovechar cabalmente las oportunidades de la globalización, lo cual ha exacerbado la marginación de África. Prestando apoyo a la conservación de la diversidad biológica de África, el uso sostenible de sus componentes y la distribución justa y equitativa de los beneficios derivados de la utilización de los recursos genéticos, en cumplimiento de los compromisos contraídos por los países en virtud de los acuerdos relacionados con la diversidad biológica en que son partes, incluidos acuerdos tales como el Convenio sobre la Diversidad Biológica y la Convención sobre el Comercio Internacional de Especies Amenazadas de Fauna y Flora Silvestre, y de conformidad con los acuerdos regionales relacionados con la biodiversidad" (Naciones Unidas, 2002: $3,53)$.

Sudáfrica y biodiversidad

De acuerdo al South África Yearbook (2011/12) - Environment, manifiesta que el país es rico en diversas especies y contiene casi el $10 \%$ del total de aves conocidas del mundo, pescado y especies de plantas, y más del $6 \%$ de mamíferos y especies de reptiles en el mundo. Con respecto a la protección de la biodiversidad, la visión del Departamento de Asuntos del Medio Ambiente es crear una próspera y equitativa sociedad que viva en armonía con los recursos con que cuenta el país.

Puerto Elizabeth Sudáfrica y biodiversidad

La Ciudad de Puerto Elizabeth tiene una población de 312, 392 habitantes comparada con la del área metropolitana al completo, que es de 1.592. 915 personas, lo que convierte a Puerto Elizabeth en unas de las ciudades más grandes de Sudáfrica. También es una de las ciudades portuarias más grandes. La temperatura de los veranos 


\section{CIÊNCIAS HUMANAS}

REVISTA DO PROGRAMA DE PÓS-GRADUAĞ̈O EM EDUCAĞ̄o

\section{(1) URI|}

de Sudáfrica (de noviembre a abril) oscila entre $\operatorname{los} 23^{\circ}$ y $\operatorname{los} 25^{\circ}$, y la temperatura en invierno es de unos $20^{\circ}$ (Ciudades.co). Al estar situado en la provincia sudafricana de Cabo del Este, Puerto Elizabeth y sus alrededores son el hogar de una magnífica variedad de vida silvestre, desde pequeños insectos y aves, con el elefante africano gigantesco. Existe una gran diversidad en las especies de fauna que se pueden encontrar aquí (ㅇ Copyright www.portelizabethforever.co.za). Como se plantea en el South África Yearbook 2011/12 - Environment, citado anteriormente, Sudáfrica se enfrenta a muchos de los problemas experimentados en los países en desarrollo donde el rápido aumento de la industrialización, el crecimiento demográfico y la urbanización ponen en peligro la calidad del medio ambiente. Por lo antes dicho, la Ciudad de Puerto Elizabeth por ser una ciudad grande, un centro portuario y comercial, en donde se desarrolla la industria, entre otros, no escapa a problemas de esta índole, y que pueden afectar al medio y específicamente a su biodiversidad.

La importancia de la Educación Ambiental en el cuidado de la biodiversidad:

La Educación Ambiental nace inicialmente del contacto con la naturaleza y del conocimiento del medio e inicia su andadura en la década de los años setenta del pasado siglo XX. Desde sus inicios quiere promover actitudes de curiosidad, respeto y valoración hacia todos los componentes del patrimonio natural. Muy pronto amplía su campo de acción al conjunto de los problemas ambientales (contaminación, aglomeraciones urbanas, consumo, residuos, cambio climático, etc.), incluyendo los aspectos socioeconómicos, (años 80) (Alcántara et al., 2006). La educación ambiental es, sin duda, uno de los medios más indicados para el rescate de valores que incluyen el respeto por la diversidad cultural y biológica. Hace ya mucho tiempo que Brasil viene entendiendo la importancia de la educación ambiental. El Ministerio de Educación elaboró en 1997 una nueva propuesta curricular conocida como Parámetros Curriculares Nacionales - PCNs, donde la dimensión ambiental pasa a ser un tema transversal en los currículos básicos de la enseñanza fundamental (de $1^{\mathrm{a}}$ a $8^{\mathrm{a}}$ años). Al cruzar todas las materias, la educación ambiental integra los temas socio-ambientales en una forma amplia y englobadora (MEC, 2000). El auge del reconocimiento de su importancia se 


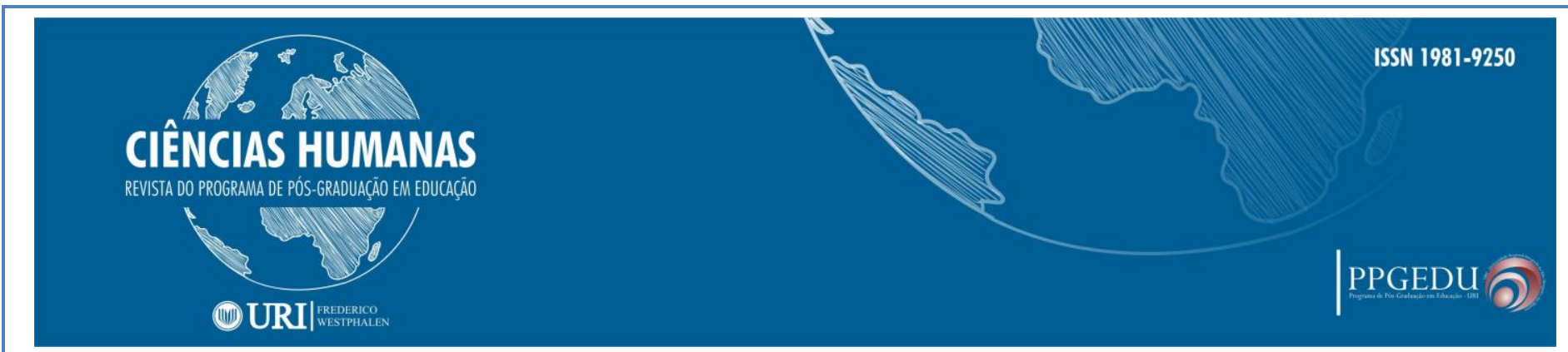

dio en abril de 1999, cuando la educación ambiental pasó a contar con la Ley $\mathrm{n}^{\mathrm{o}}$ 9795/99, que la oficializa como área esencial y permanente en todo proceso de educación del país (MEC, 2000) (Machado, 2002: 51-52). La conservación de la biodiversidad implico un cambio sustancial en el enfoque de los conservacionistas, ya que las estrategias implicaban no solamente la conservación de determinadas especies, sino la protección de ecosistemas completos que contuvieran especies importantes (Wilson, 1992: García et al., 2004: 21). La educación ambiental viene a constituir el proceso educativo que se ocupa de la relación del ser humano con su ambiente (natural y artificial) y consigo mismo, así como las consecuencias de esta relación. De esta manera, la educación ambiental debe constituir un proceso integral, que juega su papel en todo el entramado de la enseñanza y el aprendizaje. Para ello, es necesario establecer un proceso educativo que cuestione la relación de cualquier tema o actividad del ser humano, dentro de un análisis de la importancia o incidencia en la vida social y ambiental, como es la parte pedagógica y su esencia política (Martínez, 2010: 1). Es necesario contar con nuevas estrategias educativas para entender y mitigar, desde diversos puntos de vista, el deterioro ambiental de nuestro tiempo. La (EA) es la herramienta elemental para que todas las personas adquieran conciencia de la importancia de preservar su entorno y sean capaces de realizar cambios en sus valores, conducta y estilos de vida, así como ampliar sus conocimientos para impulsarlos a la acción mediante la prevención y mitigación de los problemas existentes y futuros (Sauvé, 2004:Espejel et al., 2012b: 1). Los programas ambientales (PA), conformados por un conjunto de acciones concretas y viables, diseñados y ejecutados por estudiantes, los PA son una herramienta de gran importancia y de utilidad que se deben diseñar y aplicar en las instituciones educativas para mitigar el deterioro ambiental en la escuela y en la comunidad (Espejel et al., 2012:c 2).

La (EA) o Educación para el Desarrollo Sostenible en el currículo escolar

La Educación para el Desarrollo Sostenible (EDS) permite que cada ser humano adquiera los conocimientos, las competencias, las actitudes y los valores necesarios para forjar un futuro sostenible. Educar para el desarrollo sostenible significa incorporar los 


\section{CIÊNCIAS HUMANAS}

REVISTA DO PROGRAMA DE PÓS-GRADUAĞ̈O EM EDUCAĞ̄o

\section{(1) URI|}

temas fundamentales del desarrollo sostenible a la enseñanza y el aprendizaje, por ejemplo, el cambio climático, la reducción del riesgo de desastres, la biodiversidad, la reducción de la pobreza y el consumo sostenible. Asimismo, la EDS exige métodos participativos de enseñanza y aprendizaje que motiven a los alumnos y les doten de autonomía, a fin de cambiar su conducta y facilitar la adopción de medidas en pro del desarrollo sostenible. La EDS exige cambios de gran calado en los métodos pedagógicos que se aplican actualmente (UNESCO, 2005-2014). De acuerdo a Chagollán, López y Ávila (2006), manifiesta que la Educación Ambiental (EA) esta, no es un campo de estudio como la química, física, biología, o ecología, que EA, es un proceso y que en realidad el término sería Educación para el Desarrollo Sostenible. Con esto se comprende que al desarrollar una temática, un proyecto de investigación, una unidad de aprendizaje donde se incorpore lo socio-ambiental, en ese momento se pone en práctica la EA. (Chagollán, 2006: Bedolla, 2014b).

La incorporación de lo ambiental al sistema escolarizado: Son esfuerzos para incorporar contenidos ambientales -conocimientos, actitudes, valores, y/o destrezas- a la educación formal, vía el currículum, los materiales (por ejemplo libros de texto), o los métodos de enseñanza, desde los niveles básicos de enseñanza hasta el posgrado (Nieto, 2001: 2). La incorporación de la EA en el ámbito educativo, se da a la vislumbre de lo que acontece por las problemáticas ambientales y que impactan de manera adversa en el mundo. Su planteamiento de la EA como estrategia para resarcir daños al medio ya se planteaban en las reuniones y cumbres que se realizaron antes y después de los años 70`s, como por ejemplo en la Conferencia de las Naciones Unidas sobre el Medio Humano, en Estocolmo, Suecia (1972) y que convirtió al medio ambiente en un tema de relevancia a nivel internacional. De manera específica, se plantea integrar dicha perspectiva, en la función de docencia con el propósito de que las instituciones de educación superior formen ambientalmente a los alumnos de hoy, futuros egresados, para que estén en posibilidades de detener, limitar, atenuar y/o prevenir los impactos negativos a la naturaleza en el ámbito de su acción profesional (Bravo, 2012).

De acuerdo a las aseveraciones antes dichas, se comprende que en el campo de la educación formal en el mundo, la crisis ambiental, llegó a ser una necesidad que se 


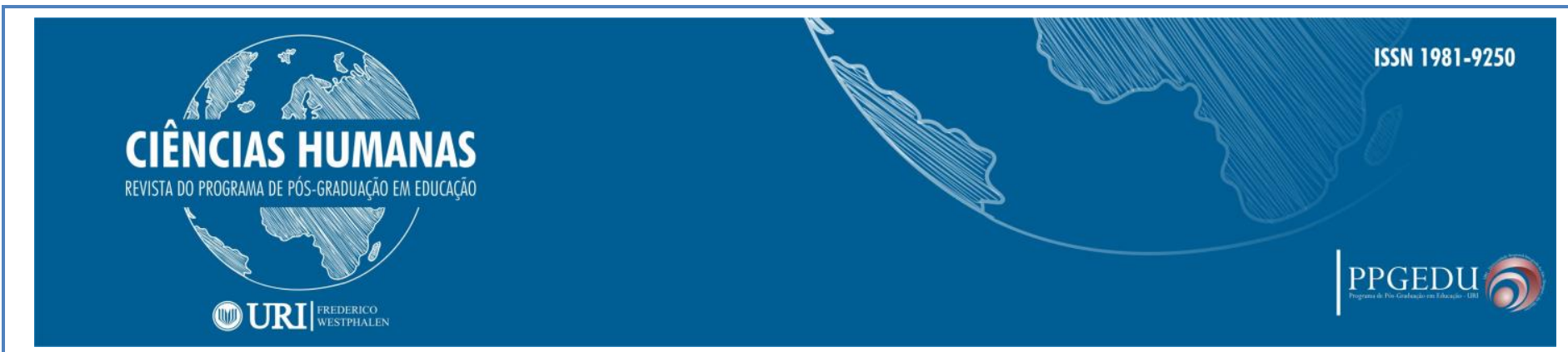

vinculó a la educación, desde el nivel básico hasta el superior. La manera de incorporarla era introducir el eje ambiental en el currículo con el fin de brindar una Educación Ambiental (EA) que promoviera cambio de conducta y aptitudes en el ser humano en pro del medio y en este caso, para preservar la biodiversidad. En el campo educativo hasta mediados de los 80 `s del siglo pasado la EA se enfocaba a sensibilizar a las personas y a promover actividades que favorecieran la conservación del ambiente "natural". En cambio, hoy en día, con el término "Desarrollo Sostenible", que tuvo su origen en el evento, las Cumbres de la Tierra -organizadas por la ONU- celebrada en Río de Janeiro en Brasil en 1992, esta paso de EA a Educación para el Desarrollo Sostenible, que comprende (Educación, sociedad, economía, medio ambiente), es decir, debemos educar considerando que lo ambiental puede repercutir en algún sector de la sociedad y no solo en la naturaleza. Como lo establece el Consejo Nacional del Ambiente de Perú (CONAM, S/F: 8-11) el concepto de ambiente ha ido evolucionando a través del tiempo y como consecuencia, los enfoques de Educación Ambiental, por lo tanto las instituciones educativas centran su atención en tres enfoques: a) Enfoque Naturalista, b) Enfoque ecologista y c) Enfoque Ambientalista. Este último enfoque, manifiesta que la Educación Ambiental concibe el ambiente como una totalidad donde todos sus elementos (incluido el ser humano) se interrelacionan sistemáticamente. Dando seguimiento a lo que estipula el CONAM, este, sugiere que la EA sea tratada como un tema transversal que impregne todo el currículo. Un currículo escolar ambientalizado es aquel que tiene contemplado el eje medio ambiente durante todas sus fases y tiene entre sus propósitos contribuir en laSustentabilidad (Bedolla et al., 2007:). Con respecto a lo dicho, puede darse el caso, que algunos planes de estudio incorporen el eje medio ambiente de manera diferente, como por ejemplo a través de una disciplina, sin embargo, este currículo no estaría completamente ambientalizado. Se concibe, pues, la E.A. no como una nueva disciplina, sino como una progresiva integración de la cuestión ambiental en todo el currículo, de acuerdo con las concepciones nórdicas, que la veían como una «dimensión». Se enfoca el medio ambiente tanto en sus aspectos físicos como sociales, culturales, económicos etc. Se recomienda también que el estudio del medio empiece en el entorno inmediato (González, 1996: 13,74). Integrar la 


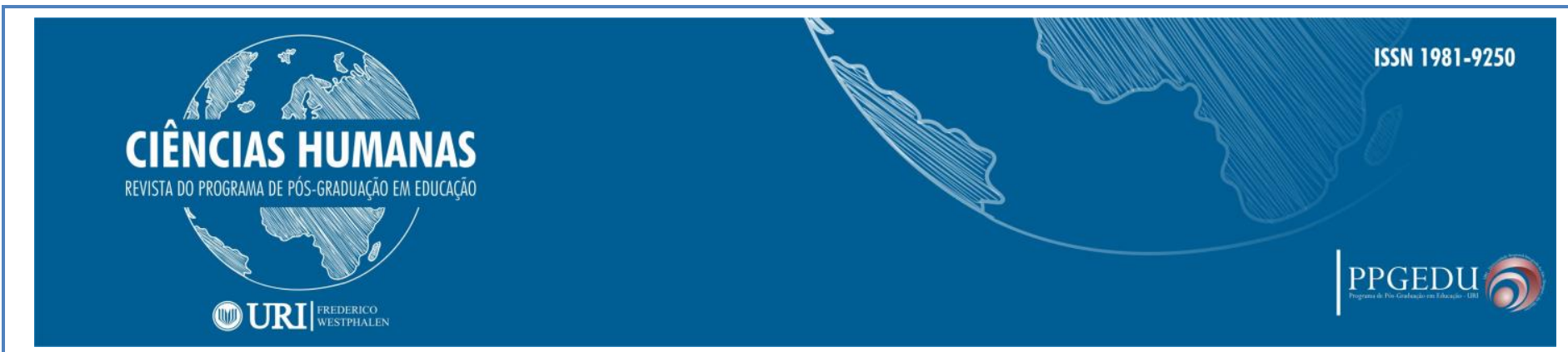

problemática ambiental dentro del contexto educativo, no debe considerarse como una añadidura más al currículo oficial, saturado ya de contenidos. Su incorporación requiere de una adecuación que integre las demandas resolutivas a la problemática ambiental como una necesidad social y como acción política, que requiere de la participación científica y transformadora de toda la sociedad (Acosta, 2000: 15-30).

\section{Materiales y Métodos}

El estudio que se presenta es una investigación de campo. Desde el punto de vista de los enfoques de investigación, este estudio es mixto, debido a que la metodología comprendida abarcó un procedimiento que va de la explicación a la descripción, la aplicación de técnicas e instrumentos, cualitativos y cuantitativos. Los diseños mixtos representan el más alto grado de combinación o integración entre los enfoques cualitativo y cuantitativo (Hernández, Fernández y Baptista (2003): Pereira, 2011: Bedolla et al., 2015: pp. 1-11). Esta investigación es descriptiva. La investigación descriptiva la llevamos a cabo cuando queremos mostrar las características de un grupo, de un fenómeno o de un sector, a través de la observación y medición de sus elementos. La información que nos proporciona un análisis descriptivo, además de ser un fin en sí mismo, la podemos utilizar como base de partida para el desarrollo de una investigación más específica (Lafuente, 2008: Bedolla et al., 2015b: pp. 1-11).

Población: La Universidad Metropolitana Nelson Mandela (NMMU), está localizada en Puerto Elizabeth Sudáfrica, su modelo de universidad, se fundamenta en un espíritu de la enseñanza y aprendizaje de los negocios para atender las prioridades regionales y nacionales, adhiriendo programas actualizados constantemente para las necesidades del mañana. La universidad es reconocida como líder en los campos, tales como, ingenierías, ecología, estudios del mar, salud comunitaria, construcción del medio ambiente, artes y diseño, contabilidad y auditoría, educación y Tecnologías de la Información. Ofrece una gran cantidad de programas académicos y profesionales desde licenciaturas hasta posgrados. Los siete programas académicos que son ofrecidos en los siete campos son; Artes, Negocios y Ciencias Económicas, Educación, Ingenierías (construcción del medio ambiente y Tecnologías de la Información), Ciencias de la 


\section{CIÊNCIAS HUMANAS}

REVISTA DO PROGRAMA DE PÓS-GRADUAĞ̈O EM EDUCAĢĀO

\section{(10)URI|}

Salud, Derecho y Ciencias (NMMU, 2016a: 2,36-39). Respecto a su planta de estudiantes, esta universidad cuenta con alrededor de 26,347, de los cuales 12,613 son mujeres y 13, 734 son hombres. De $\operatorname{los} 26,347,22,558$ son estudiantes de licenciatura, considerando ello, 10,792 hombres y 11, 766 mujeres. En este tenor, son de posgrado, 3, 789 de los cuales 1,821 son mujeres y 1968 son hombres. Otras características de los estudiantes, se aprecian en las tablas presentadas. Respecto a la planta de académicos, la universidad cuenta con un total de 1652, entre permanentes y temporales. Ver tabla, I, II y III.

Tabla I: Headcount per Facultyfor 2015 (Plantilla por facultad del año 2015).Matricula de estudiantes por facultad.Universidad Metropolitana Nelson Mandela (NMMU) Puerto Elizabeth Sudáfrica.

\begin{tabular}{|c|c|c|c|c|c|c|c|}
\hline Faculty Name & COUNT & WHITE & $\begin{array}{r}\text { COLOURE } \\
\text { D }\end{array}$ & $\begin{array}{r}\text { INDIA } \\
\mathbf{N}\end{array}$ & BLACK & MALES & $\begin{array}{l}\text { FEMALE } \\
\text { S }\end{array}$ \\
\hline ARTS & 3288 & 868 & 446 & 62 & 1912 & 1290 & 1998 \\
\hline $\begin{array}{l}\text { BUSINESS AND ECONOMIC } \\
\text { SCIENCES }\end{array}$ & 9487 & 1467 & 1327 & 146 & 6547 & 4364 & 5123 \\
\hline $\begin{array}{l}\text { DVC RESEARCH AND } \\
\text { ENGAGEMENT }\end{array}$ & 301 & 278 & 3 & 0 & 20 & 112 & 189 \\
\hline EDUCATION & 1779 & 755 & 545 & 29 & 450 & 548 & 1231 \\
\hline $\begin{array}{l}\text { ENGINEERING,BUILT } \\
\text { ENVIRON \& IT }\end{array}$ & 4179 & 984 & 456 & 86 & 2653 & 3172 & 1007 \\
\hline HEALTH SCIENCES & 3368 & 698 & 546 & 76 & 2048 & 1006 & 2362 \\
\hline LAW & 1173 & 208 & 166 & 26 & 773 & 571 & 602 \\
\hline SCIENCE & 2772 & 948 & 205 & 56 & 1563 & 1550 & 1222 \\
\hline & 26347 & 6206 & 3694 & 481 & 15966 & 12613 & 13734 \\
\hline
\end{tabular}

Fuente: Dr. LogamurthieAthiemoolam, Profesor de la Facultad de Educación de la NMMU.

Tabla II: Matricula de estudiantes de la Universidad Metropolitana Nelson Mandela (NMMU) Puerto Elizabeth Sudáfrica. UG: Undergraduate: de licenciatura, PG: Posgraduate: de posgrado.

\begin{tabular}{|l|l|l|l|r|l|l|l|}
\hline UG/PG & \multicolumn{1}{|c|}{ COUNT } & \multicolumn{1}{|c|}{ WHITE } & \multicolumn{1}{|c|}{ COLOURED } & INDIAN & BLACK & MALES & FEMALES \\
\hline $\mathrm{U}$ & 22558 & 5112 & 3249 & 384 & 13813 & 10792 & 11766 \\
\hline $\mathrm{P}$ & 3789 & 1094 & 445 & 97 & 2153 & 1821 & 1968 \\
\hline & 26347 & 6206 & 3694 & 481 & 15966 & 12613 & 13734 \\
\hline
\end{tabular}




\section{CIÊNCIAS HUMANAS}

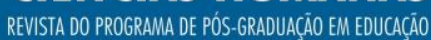

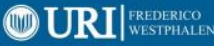

Tabla III. Current Academic Staff Per Faculty (Personal Académico actual por facultades en la NMMU.P: Permanent, T: Temporal.

\begin{tabular}{|l|l|l|l|} 
& \multicolumn{3}{l}{$\begin{array}{l}\text { PERMANENT OR TEMP } \\
\text { (Total) }\end{array}$} \\
\hline FACULTY & P & T & Total \\
\hline ARTS & 103 & 89 & 192 \\
\hline $\begin{array}{l}\text { BUSINESS AND ECONOMIC } \\
\text { SCIENCES }\end{array}$ & 130 & 81 & 211 \\
\hline DVC RESEARCH AND ENGAGEMENT & 5 & 120 & 125 \\
\hline EDUCATION & 44 & 41 & 85 \\
\hline ENGINEERING,BUILT ENVIRON \& IT & 84 & 34 & 118 \\
\hline GEORGE CAMPUS & & 1 & 1 \\
\hline HEALTH SCIENCES & 87 & 33 & 120 \\
\hline LAW & 28 & 11 & 39 \\
\hline REGISTRAR & & 527 & 527 \\
\hline SCIENCE & 139 & 90 & 229 \\
\hline SD COMM \& STAKEHOLDER LIAISON & & 2 & 2 \\
\hline TEACHING AND LEARNING & 3 & & 3 \\
\hline & 623 & 1.029 & 1.652 \\
\hline
\end{tabular}

Fuente: Dr. LogamurthieAthiemoolam, Profesor de la Facultad de Educación de la NMMU.

Muestra: Este estudio se realizó en la Facultad de Educación dependiente de la (NMMU), en las instalaciones localizadas en Puerto Elizabeth. Esta facultad tiene tres áreas de formación, entre las que se encuentran, Escuela para Maestros de la Educación de nivel principiante, escuela para el desarrollo continuo y profesional. Escuela de educación para el compromiso y la investigación. Esta investigación se realizó en esta facultad en el área Escuela para maestros de la Educación de nivel principiante (NMMU,2016b: 2,36-39). El total de estudiantes con que cuenta esta facultad es de 1779 de los cuales, 548 son hombres y 1231 son mujeres. Con respecto a que esta institución cuenta con tres áreas, como anteriormente se menciona, la escuela para el desarrollo continuo y profesional cuenta con un total de 137 de los cuales 42 son hombres y 95 son mujeres, la Escuela de educación para el compromiso y la investigación, cuenta con un total de 98 estudiantes de los cuales, 39 son hombres y 59 son mujeres y en el área Escuela para Maestros de la Educación de nivel principiante, cuenta con un total de 1544 de los cuales 467 son hombres y 1077 son mujeres (Ver tabla IV). Respecto a la planta de académicos, esta facultad cuenta con 85 profesores de 


\section{CIÊNCIAS HUMANAS}

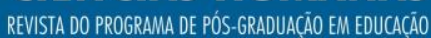

\section{(1) URI|}

los cuales, 44 son permanentes y 41 son temporales. Para el muestreo se recurrió al enfoque cualitativo (Ver tabla V). La muestra en el proceso cualitativo es un grupo de personas, eventos, sucesos, comunidades, etc., sobre el cual se habrán de recolectar los datos, sin que necesariamente sea representativo del universo o población que se estudia (Hernández et al 2008, p.562: Angulo, 2011: Bedolla et al., 2015c: pp. 1-11).). El tamaño de la muestra se determinó en función del tipo de muestreo elegido, de la precisión deseada, de los recursos económicos, de los tiempos con los que contemos, y de la facilidad de selección (Lafuente, 2008b: :Bedolla et al., 2015d: pp. 1-11). Fueron alrededor de 46 estudiantes seleccionados y diez maestros. Con los datos proporcionados se logró tener información valiosa para cumplir con los objetivos del presente estudio. Se reitera que la muestra se seleccionó cualitativamente, el estudio en cuestión es mixto.

Tabla IV. Estudiantes inscritos en la Facultad de Educación de la NMMU.

\begin{tabular}{|c|c|c|c|c|c|c|c|c|}
\hline Acad Year & Department Name & $\begin{array}{l}\text { EnrolledS } \\
\text { tudents }\end{array}$ & White & Coloured & Indian & African & Males & Females \\
\hline \multirow[t]{17}{*}{2015} & $\begin{array}{l}\text { SCHOOL FOR } \\
\text { CONTINUING PROF } \\
\text { DEV }\end{array}$ & 1 & 0 & 0 & 0 & 1 & 0 & 1 \\
\hline & & 3 & 0 & 1 & 0 & 2 & 0 & 3 \\
\hline & & 1 & 0 & 1 & 0 & 0 & 0 & 1 \\
\hline & & 25 & 14 & 3 & 0 & 8 & 4 & 21 \\
\hline & & 85 & 21 & 29 & 3 & 32 & 33 & 52 \\
\hline & & 2 & 0 & 0 & 0 & 2 & 0 & 2 \\
\hline & & 1 & 0 & 0 & 0 & 1 & 0 & 1 \\
\hline & & 5 & 0 & 1 & 0 & 4 & 3 & 2 \\
\hline & & 1 & 0 & 0 & 0 & 1 & 1 & 0 \\
\hline & & 13 & 6 & 5 & 0 & 2 & 1 & 12 \\
\hline & $\begin{array}{l}\text { SCHOOL FOR ED } \\
\text { RESEARCH \& ENGAG }\end{array}$ & 11 & 5 & 2 & 0 & 4 & 2 & 9 \\
\hline & & 40 & 13 & 14 & 2 & 11 & 17 & 23 \\
\hline & & 1 & 0 & 0 & 0 & 1 & 0 & 1 \\
\hline & & 1 & 0 & 0 & 0 & 1 & 1 & 0 \\
\hline & & 18 & 5 & 0 & 1 & 12 & 6 & 12 \\
\hline & & 27 & 3 & 10 & 1 & 13 & 13 & 14 \\
\hline & $\begin{array}{l}\text { SCHOOL FOR INITIAL } \\
\text { TEACHER ED }\end{array}$ & 8 & 4 & 3 & 0 & 1 & 3 & 5 \\
\hline
\end{tabular}




\section{CIÊNCIAS HUMANAS}

REVISTA DO PROGRAMA DE PÓS-GRADUAĞ̈O EM EDUCAĢ̄o

\section{(10)URI|}

\begin{tabular}{|l|l|l|l|l|l|l|l|l|}
\hline & 1 & 0 & 0 & 0 & 1 & 0 & 1 \\
\hline & 370 & 240 & 81 & 9 & 40 & 1 & 369 \\
\hline & 277 & 89 & 124 & 2 & 62 & 87 & 190 \\
\hline & 356 & 193 & 102 & 7 & 54 & 129 & 227 \\
\hline & 86 & 32 & 48 & 1 & 5 & 33 & 53 \\
\hline & 382 & 110 & 107 & 3 & 162 & 195 & 187 \\
\hline & & 64 & 20 & 14 & 0 & 30 & 19 & 45 \\
\hline & & 1779 & 755 & 545 & 29 & 450 & 548 & 1231 \\
\hline
\end{tabular}

Fuente: Dr. LogamurthieAthiemoolam, Profesor de la Facultad de Educación de la NMMU.

Tabla V. Planta de académicos, permanentes y temporales en la Facultad de Educación de la NMMU.

\section{PERMANENT OR TEMP (Total)}

\section{FACULTY}

EDUCATION

\begin{tabular}{|l|l|l|}
\hline $\mathbf{P}$ & $\mathbf{T}$ & Total \\
\hline
\end{tabular}

$44 \quad 41$

Fuente: Dr. Logamurthie Athiemoolam, Profesor de la Facultad de Educación de la NMMU.

Técnicas e instrumentos empleados: Se diseñaron dos instrumentos para recolectar datos. Estos fueron basados en la encuesta, la cual se diseñó y elaboró en español y traducida al inglés debido a que en el área de estudio es este idioma el que se habla. Se aplicó a estudiantes y a docentes. El objetivo de este instrumento fue identificar la importancia de la Educación Ambiental en el cuidado para preservar la biodiversidad a través del currículo y desde la perspectiva de los estudiantes y los profesores de la Facultad de Educación perteneciente a la Universidad Metropolitana Nelson Mandela. La variable de la encuesta aplicada a estudiantes y docentes, consideró dos elementos (conocimientos generales), este primer elemento enmarcó (problemática ambiental, la Educación Ambiental y la biodiversidad), el segundo, (la implementación de la Educación Ambiental para la biodiversidad en el currículo escolar). Los instrumentos se aplicaron durante el mes de noviembre del año 2015. La encuesta fue procesada a través del programa estadístico IBM SPSS Statistics 20, se tuvieron datos cuantitativos con su procesamiento, sin embargo, dichos datos fueron traducidos cualitativamente. Los resultados mostraron lo siguiente:

\section{RESULTADO}




\section{CIÊNCIAS HUMANAS}

Información de los estudiantes

Se inician los resultados con la información proporcionada por los estudiantes: Como se menciona en la muestra, fueron cuarenta y seis estudiantes seleccionados, a ellos les fue aplicado el instrumento que se menciona en el punto de técnicas e instrumentos empleados. Por ser una investigación mixta que concierne lo cualitativo los resultados se describen de manera cualitativa. Casi todos los jóvenes cursan el segundo semestre de la Facultad de Educación, en el área Escuela para Maestros de la Educación de nivel principiantes, pocos fueron de semestres más avanzados, la mayor parte de la población fueron mujeres solo doce fueron hombres. La mayoría son de Puerto Elizabeth, algunos vienen de otros países de África e incluso de países fuera de este continente. Casi todos comprenden que la problemática ambiental se hace presente en todo el mundo, la mitad de ellos, hace referencia a que últimamente la Educación Ambiental ha cobrado relevancia en el sentido de preservar el medio, enfocándose solamente en la subsistencia del hombre, pero olvidándose del mantenimiento de la biodiversidad, como por ejemplo de las plantas y animales, por esta razón, casi todos aseguran que el ser humano necesita promover un sentido analítico, crítico y responsable ante el cuidado de la diversidad biológica. Casi todos están de acuerdo en que los niveles culturales de la persona impactan en su bienestar, por tal motivo, casi todos están de acuerdo en que la Educación Ambiental es un proceso que coadyuva en la promoción de un sentido crítico y responsable para el cuidado y bienestar de recursos que la naturaleza nos ofrece. Casi todos aseguran que África es un continente rico en biodiversidad (plantas y animales), en el mismo tenor, que aquí se encuentra establecida una gran diversidad de grupos étnicos y más de la mitad asegura que estas civilizaciones no agreden por completo al medio. Más de la mitad asegura que los campesinos, ganaderos o algunas otras personas, afectan de alguna forma las áreas ecológicas, por alguna de necesidad de subsistencia, sin embargo más de la mitad asegura que eso no impacta en el deterioro ambiental. En Puerto Elizabeth Sudáfrica y en sus alrededores, se puede observar una gran amplia variedad de especies vegetales y animales, dice la mayoría, sin embargo más de la mitad asegura que son escasos los valores sociales y 


\section{CIÊNCIAS HUMANAS}

REVISTA DO PROGRAMA DE PÓS-GRADUAĞ̈O EM EDUCAĞ̄o

\section{(1) URI|}

culturales, provistos para la protección de la diversidad biológica. En una segunda pregunta relacionada con valores y respeto, se fortalece que estos son escasos, sin embargo menos de la mitad mencionan que si se promueven. Casi todos aseguran que mantener y preservar la biodiversidad es el camino para promover la sustentabilidad que se requiere. En relación a una legislación para proteger la biodiversidad, poco más de la mitad dijo estar de acuerdo, sin embargo casi muchos dijeron lo contrario, esto da a comprender que desconocen si existe o no una ley que regule esta situación.

En relación al currículo universitario y específicamente a la carrera que cursan, más de la mitad consideran que el currículo o plan de estudio de la carrera que cursan, no plantea la importancia de la Educación Ambiental en el cuidado de la biodiversidad. Muy pocos los que dicen lo contrario. La mayoría dice que las materias o unidades de aprendizaje planteadas en el currículo, no integran competencias sobre Educación Ambiental o bien sobre el tema en cuestión. Sin embargo hubo algunos que si lo afirman. De igual manera más de la mitad, asegura que sus profesores en las clases no promueven el respeto y cuidado por la biodiversidad que se requiere. Menos de la mitad, dicen lo contrario de lo dicho. Más de la mitad afirman que sus profesores no cuentan con las competencias en temas de protección y cuidado del medio ambiente y su relación con la sustentabilidad. Casi la mayoría afirma que el docente, no pide a sus estudiantes promover una cultura de respeto a plantas y animales., pero casi todos afirman que los recursos, agua, aire, suelo y energía, deben ser usados racionalmente para beneficiar la biodiversidad y que comprenden que la situación ambiental actualmente atraviesa por una serie de problema, causas y consecuencias. El haber preguntado, si en la institución donde estudian, el agua es reciclada y usada para las plantas, etc. casi todos dijeron que no. Tal vez esto se debe a falta de conocimiento o efectivamente a que no se emplea la práctica del reciclaje, si es así, esto indica, la falta de Educación Ambiental. Sin embargo la mayoría, analiza críticamente y piensa en propuestas de solución del cuidado ambiental y promueve valores para proteger la biodiversidad, de la misma manera, consideran que es necesario individual y colectivamente diseñar propuestas o soluciones a aquello que afecte la sustentabilidad del entorno. Casi todos están afirman que en la institución (en su escuela), los temas 


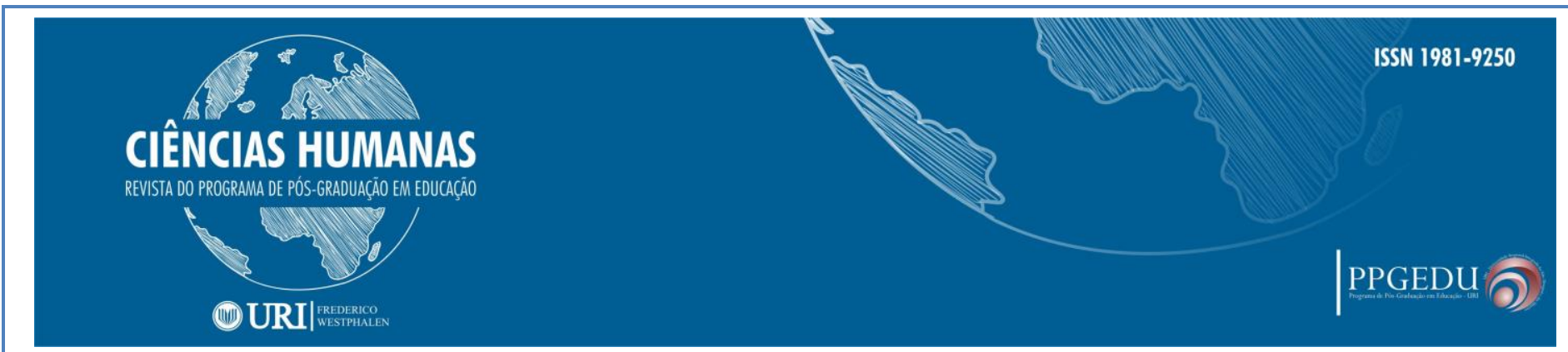

ambientales no son discutidos y las acciones no son tomadas en cuenta para proponer programas de residuos sólidos, que aquí se generan. Poco más de la mitad, dijeron que se comprometen participar en proyectos que apoyen en el mejoramiento y protección del medio, fueron muchos los que presentan resistencia en proyectos de esta naturaleza. Finalmente, más de la mitad dicen no poner en práctica en su vida diaria, lo que aprenden en la escuela acerca del medio ambiente y la biodiversidad.

Información de los docentes

Respecto a la información proporcionada por los profesores, se informa que, fueron diez maestros encuestados, de los cuales cinco son hombres y cinco mujeres, casi todos los encuestados mencionan que son de Puerto Elizabeth, uno de ellos dijo que es de otro lugar. Todos comprenden que la problemática ambiental se hace presente en todo el mundo, de igual manera que la importancia de la Educación Ambiental se enfoca a la subsistencia del individuo y hace a un lado la biodiversidad y que los seres humanos necesitan promover un sentido crítico y responsable en el cuidado de la biodiversidad. La mayoría están de acuerdo en que los niveles culturales del individuo contribuyen en el mejoramiento de la diversidad biológica. Casi todos consideran que la Educación Ambiental, contribuye en la promoción de un sentido crítico, analítico y responsable sobre el uso y preservación de los recursos y en el mismo tenor hacen referencia que África es un continente rico en biodiversidad (plantas, animales y ecosistemas). La mayoría dice que África es un lugar en donde hay una variedad de grupos étnicos y que estos grupos, no afectan la naturaleza, sin embargo casi todos afirman que los granjeros, campesinos, etc. están haciendo uso de las áreas ecológicas para su modo de subsistencia. Las influencias de otras culturas, estilos de vida, etc. no contribuyen en el mejoramiento del medio dijo la mayoría, casi todos dicen que en Puerto Elizabeth y sus alrededores se puede observar una gran variedad de flora y fauna. Más de la mitad, menciona que los valores sociales y la cultura es escasa en las personas de este lugar, para la sustentabilidad de la biodiversidad. Casi todos, dicen que en este lugar no se promueve el respeto por la biodiversidad cultural y biológica, sin embargo todos están de acuerdo en que si se promueve, se contribuiría al desarrollo sustentable. 


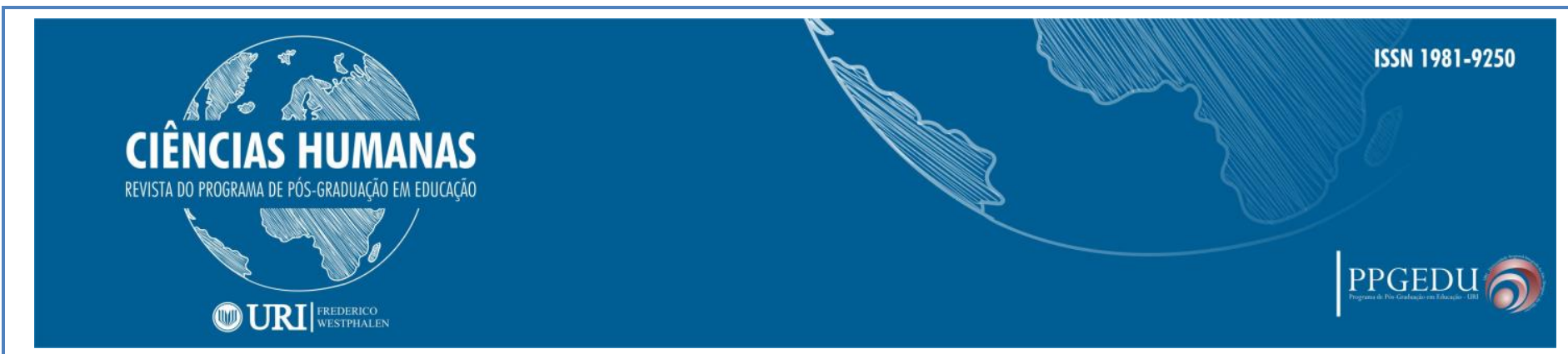

Poco más de la mitad afirma que el país cuenta con una legislación en materia ambiental para proteger la biodiversidad. Al preguntar a cerca de la implementación de la Educación Ambiental (EA) en el cuidado de la biodiversidad en el currículo o plan de estudio en la facultad que laboran, casi todos dijeron que esto no está presente, un poco más de la mitad, mencionaron que la biodiversidad no es un tema emergente que debe ser integrado en los planes de estudio en África y principalmente en Puerto Elizabeth, muy pocos, dijeron estar de acuerdo. Casi todos afirmaron que los objetivos de las materias que integra el currículo no consideran competencias (conocimientos, habilidades y valores), para proteger la biodiversidad. Poco más de la mitad de los docentes afirma contar con las competencias en temas de protección y cuidado del medio ambiente para coadyuvar en la sustentabilidad, hubo algunos que dijeron no contar con ellas. La mayoría menciona que no diseñan estrategias didácticas para promover la educación ambiental que contribuyan a la protección de la biodiversidad en el proceso de enseñanza-aprendizaje. La mayor parte de estos profesores, piden a sus estudiantes, apagar la luz, el aire acondicionado, poner la basura en su lugar, favoreciendo de una forma a la biodiversidad. Todos dicen que en la Universidad Nelson Mandela se promueven programas para proteger la biodiversidad. Más de la mitad asegura que no se promueve el respeto y cuidado de la diversidad biológica y cultural. Todos los docentes no realizan actividades que tengan relación con la preservación de la biodiversidad, sin embargo poco más de la mitad, considera importante que los estudiantes desarrollen competencias para preservar la biodiversidad y contribuir en la sustentabilidad que se requiere, de igual manera, comentaron que no se trabaja en proyectos que tengan como fin los aprendizajes para fomentar la responsabilidad en los estudiantes a cerca de esta temática. Casi todos afirman que el medio ambiente y temas de biodiversidad no se plantean en el currículo trasversalmente, de igual manera, mencionan que no están seguros si en la escuela, exista un programa que administre los residuos sólidos en la universidad, sin embargo, al preguntar si la universidad tiene una regulación interna para la protección de la biodiversidad, más de la mitad contestaron estar de acuerdo. 


\section{CIÊNCIAS HUMANAS}

REVISTA DO PROGRAMA DE PÓS-GRADUAĞ̈O EM EDUCAĞ̄o

\section{(10)URI|}

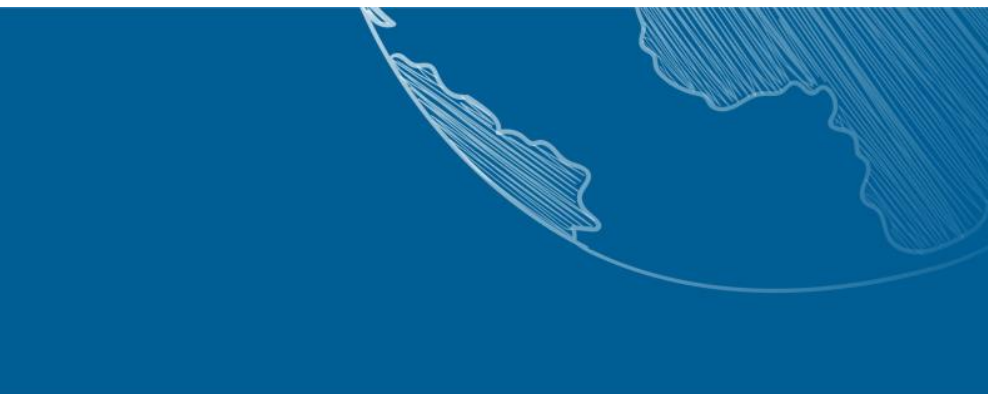

\section{DISCUSIÓN Y CONCLUSIONES}

La crisis ambiental que enfrenta nuestro planeta es sin duda un tema sobresaliente, que urgentemente debe ser atendido por toda la sociedad en su conjunto, grupos u organizaciones civiles, los gobernantes, las instituciones, principalmente las educativas (no importando el nivel que imparta), etc. La universidad, en este caso, no debe ser la excepción, estas, deben de diseñar estrategias que permitan que la (EA) sea vista desde este espacio, como una respuesta a exigencias en esta materia y en si, como una alternativa que contribuya a minimizar, corregir y dar propuestas de solución a las problemáticas ambientales presentes y que principalmente están causando estragos en la biodiversidad que el mundo requiere para su subsistencia. La importancia de la Educación para el Desarrollo Sostenible o (EA) radica entonces en las acciones que se ponen en marcha para el mantenimiento y preservación de la biodiversidad y de los recursos naturales. En el campo educativo, ello se refleja en el currículo de una institución y por ende, en sus acciones programadas, el éxito de estas últimas, depende del grado y nivel de realización y aplicación de la institución y sus actores. Este trabajo presenta los resultados de una investigación realizada en la Facultad de Educación de la Universidad Metropolitana Nelson Mándela en Puerto Elizabeth Sudáfrica, que tuvo el propósito de dar a conocer la importancia que se da a la Educación Ambiental para la preservación de la biodiversidad, para lo cual tomó en consideración, puntos de vista de estudiantes y docentes. La institución mencionada está ubicada en un país que se caracteriza por poseer una gran diversidad de flora y fauna en el continente africano. De manera general, los resultados reflejan que esta facultad, da una mínima importancia a la (EA) para el cuidado de la diversidad biológica, esto se constató en el análisis y resultado de la presente.

La necesidad de incorporar la (EA) en el ámbito educativo y formal no fue apenas, ya tiene algunas décadas, sin embargo en algunos países esto fue posterior. En México, la Educación Ambiental (EA) tuvo un inicio tardío respecto a otros países norteamericanos y europeos (González et al., 2000:3). Con esta aseveración se comprende que en países de África, la introducción de la (EA) en el ámbito educativo fue similar al caso de México. Sin embargo en ese continente y específicamente en 


\section{CIÊNCIAS HUMANAS}

REVISTA DO PROGRAMA DE PÓS-GRADUAĞ̈O EM EDUCAĞ̄o

\section{(10) URI|}

Johannesburgo Sudáfrica en el año 2002, se celebró la Cumbre Mundial sobre el Desarrollo Sostenible, en dicha cumbre trataron acciones sustentables para el mundo y para el continente africano y con respecto a la (EA), a esta se le incorporó en ese entonces el término "sustentable". La nueva conceptualización de la EAS de Johannesburgo - bajo la orientación de la incorporación del desarrollo sustentable a la educación superior (Bravo, 2012b). Esto indica ligar lo natural con lo social. De acuerdo a (Nieto et al., 2008), la Educación Ambiental para la Sustentabilidad (EAS) implica relacionar (ambiente, economía, sociedad, política, naturaleza, cultura). La cumbre sobre el desarrollo sostenible, realizada en dicho país africano, reforzó la estrategia de implementar acciones en pro del medio ambiente y en busca de un mundo y un África sostenible y en materia de (EA), esta, se perfiló como el medio para lograr dicha sustentabilidad que se requiere, a partir de ese momento, ese fue el método para incorporarla al campo educativo. Teniendo como fundamento las problemáticas existentes que suceden en el mundo y especialmente en el continente africano y tal como lo establecen las (Naciones Unidas, 2002c: 3,53). "el desarrollo sostenible ha seguido siendo difícil de alcanzar para muchos países de África ”y el South África Yearbook 2011/12 - Environment que estipula que "Sudáfrica se enfrenta a muchos de los problemas experimentados en los países en desarrollo donde el rápido aumento de la industrialización, el crecimiento demográfico y la urbanización ponen en peligro la calidad del medio ambiente. Lo antes dicho, hace cuestión al porque en la facultad de educación donde se realizó la investigación no se da importancia a la (EA) al menos para preservar los recursos naturales, la dimensión ambiental no aparece en el currículo y por lo tanto los docentes y estudiantes cuentan con mínimas competencias al respecto. África posee una gran biodiversidad y como lo estipula el documento citado (South África Yearbook 2011/12), Sudáfrica posee una gran diversidad biológica y representativa en el mundo, al igual el documento (@) Copyright www.portelizabethforever.co.za), expresa que en Port Elizabeth existen biomas o comunidades naturales donde existen plantas y animales, volviendo a la pregunta anterior y la universidad que hace al respecto para preservar esta biodiversidad. La falta de conciencia y cultura de las personas, la industrialización, la sobrepoblación, etc. 


\section{CIÊNCIAS HUMANAS}

REVISTA DO PROGRAMA DE PÓS-GRADUAĞ̈O EM EDUCAĞ̄o

\section{(1) URI|}

pueden terminar con este recurso. El ser humano es parte de la naturaleza y por lo tanto debe promover un sentido crítico, analítico y reflexivo y de ahí considerar que si la naturaleza se debilita, ello impactará negativamente en lo social, lo económico, la salud, etc.

En esta investigación, se aplicó una encuesta a estudiantes y docentes, consideró dos elementos (conocimientos generales), este primer elemento enmarcó (problemática ambiental, la Educación Ambiental y la biodiversidad), el segundo, (la implementación de la Educación Ambiental para la biodiversidad en el currículo escolar).

Con respecto a los estudiantes, y en relación a la comprensión de la problemática ambiental en el mundo, la EA y la biodiversidad, ellos comprenden de alguna manera que la problemática ambiental, se hace presente en el mundo, pero que las acciones que se realizan en materia de EA, solo están orientadas a preservar la existencia humana, con esto se cita una vez más lo establecido en El Informe de la Cumbre Mundial sobre el Desarrollo Sostenible, "La pobreza sigue siendo un gran obstáculo y la mayoría de los países del continente no han podido aprovechar cabalmente las oportunidades de la globalización, lo cual ha exacerbado la marginación de África" (Naciones Unidas, 2002d: 3,53). Con ello, se percibe que los programas sociales y que tienen que ver con lograr el desarrollo sustentable, están relacionados con la pobreza, hambre, desnutrición, salud entre otros y hacen a un lado el medio ambiente. La prioridad es el ser humano. La (EA) para proteger la biodiversidad de su contexto pasa a un segundo o tercer plano. Los estudiantes que dan esta información son provenientes del lugar donde está ubicada la universidad, así como de otros países de África e incluso países fuera del continente. Todos los estudiantes afirman que el nivel cultural de la persona impacta en el cuidado y bienestar que la naturaleza nos ofrece. Es importante citar en esta línea a (Nieto et al. 2008b) ya que establece que en el diseño de un currículo es importante considerar el proceso de contextualización. Seria en esta parte en donde los elaboradores del currículo tomen en cuenta que las problemáticas ambientales y las necesidades de los estudiantes, se han tomadas en cuenta en su diseño. Al incorporar el eje medio ambiente, ello implica desarrollar el proceso de la EA, ya sea como una disciplina o de forma transversal como lo establece el CONAM en líneas de este mismo documento. África es 


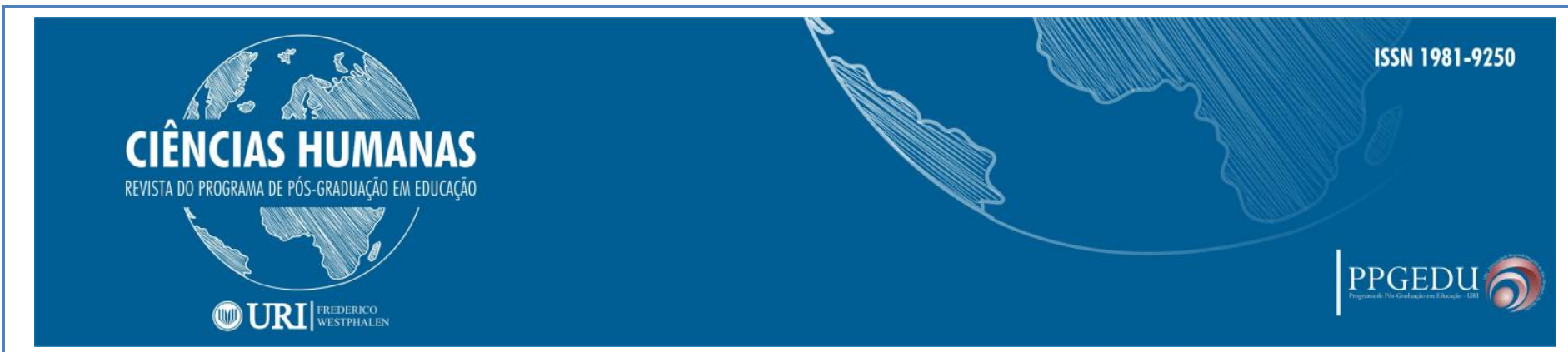

un país rico en biodiversidad y por consiguiente Sudáfrica, por lo tanto, no hay obstáculos para impedir que la EA no se desarrolle en el programa educativo donde se realizó la investigación. Los estudiantes afirman que en el contexto de esta región se afectan las áreas ecológicas y sin embargo manifiestan que ello no impacta en el deterioro ambiental. Los jóvenes afirman existe la falta de valores y cultura para la protección de la biodiversidad biológica, muchos no conocen si existen regulaciones gubernamentales en pro de la biodiversidad. Esto es la prueba de que los jóvenes no están siendo críticos en el sentido de cuidar la naturaleza y por ende la biodiversidad.

Continuando con los estudiantes y considerando la segunda variable (la implementación de la Educación Ambiental para la biodiversidad en el currículo escolar). El elemento medio ambiente no está presente en el plan de estudio de la "Escuela para maestros de la Educación de nivel principiante" de la Facultad de Educación de la Universidad Metropolitana Nelson Mandela en Puerto Elizabeth Sudáfrica y por lo tanto la (EA) para la protección de la biodiversidad también. Las materias, ni los profesores no tocan temas relacionados con EA para preservar la naturaleza, manifiestan los alumnos. Las actividades que se realizan solo son para gestionar competencias en el campo de la formación profesional, en este caso, para formar competencias docentes por ser un programa de esta índole. Aunado a que el currículo no contempla esta vertiente, por lógica los profesores no cuentan con las competencias, ni tampoco las estrategias para desarrollar un tema apegado a preservar la biodiversidad y del mismo modo entonces los estudiantes solo desarrollan conocimientos, habilidades y valores apegados a sus competencias específicas. Hay jóvenes que si se interesan en que la EA para la protección de la diversidad biológica sea incorporada en el currículo, sin embargo, también hubo jóvenes que le interesa solamente formar competencias en el área de la docencia y ya. Introducir el elemento medio ambiente (trasversalmente) en el currículo no es adherirle materias o disciplinas al currículo ya establecido, es impregnar el mismo de este elemento, para que en cada disciplina se busque la relación de su objetivo y dicho elemento, con la finalidad de desarrollar la EA para la biodiversidad y así se contribuya a la sustentabilidad. 


\section{CIÊNCIAS HUMANAS}

REVISTA DO PROGRAMA DE PÓS-GRADUAC̄̄o EM EDUCACĞ̄o

(1) URI|

Respecto a los profesores, todos están de acuerdo a que la problemática ambiental se hace presente en todo el mundo y que la (EA), se orienta a la subsistencia del individuo. Esta afirmación fue la misma que dan los estudiantes y como se dijo anteriormente, en África, la prioridad es el ser humano. El medio ambiente y por ende la biodiversidad va un segundo o tercer plano. África y en especial Sudáfrica es rico en biodiversidad, sin embargo las personas no cuidan la naturaleza, a falta de valores y cultura, estos, van de la mano con la educación y si esta, no se promueve ni en la casa ni en la escuela corre el riesgo, de que seamos inconscientes de lo que sucede en nuestro entorno. Los maestros afirmaron de nueva cuenta al igual que los estudiantes, que el plan de estudio de la institución donde se realizó la investigación no está contemplada la (EA) para el cuidado de la biodiversidad, y que dicho tema no es emergente, por lo tanto, no tiene que aparecer en el currículo ni como materia ni tampoco como un tema transversal que impregne todo el programa. Esto contradice la necesidad de implementar la (EA) en la Educación superior, como se ha discutido en las reuniones y cumbres mundiales que tienen que ver con medio ambiente y como lo fue en la cumbre de Johannesburgo en el 2002 que a la (EA), se le adhirió el término sustentabilidad. "Integrar dicha perspectiva, en la función de docencia con el propósito de que las instituciones de educación superior formen ambientalmente a los alumnos de hoy" (Bravo, 2012b). Las afirmaciones de los docentes, distan mucho de lo estipulado en los acuerdos mundiales y por la razón de que un currículo debe de considerar un estudio de su contexto. Por el hecho de que el currículo no contempla la dimensión ambiental, a la biodiversidad no se le da atención, y por ende los programas de unidades de aprendizaje no enlazan temas para preservar la biodiversidad biológica; sin embargo, hubo docentes que manifiestan que cuentan con competencias ambientales, pero no desarrollan estrategias de esta índole en el aula. Esto, está aunado a los objetivos del currículo, es decir, si el currículo, lo contemplará, se pondría en marcha el proceso de (EA) para la biodiversidad. No todo es malo, a pesar de que no se contempla el cuidado del medio de manera oficial, los profesores apoyan de algún modo con alguna actividad práctica en favor del mismo, ordenando la basura, apagando la luz cuando no se ocupa, etc. Algo muy importante que comentaron todos los profesores, es que en la Universidad Nelson 


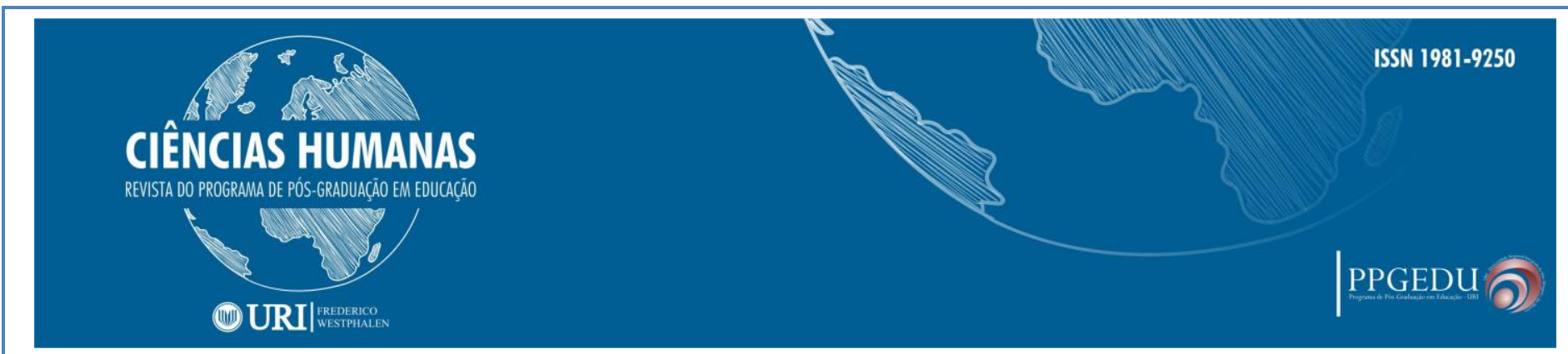

Mandela si se promueven los programas para proteger la biodiversidad, esto a raíz, de que la institución cuenta con una facultad denominada (construcción del medio ambiente), ciertamente, esta facultad orienta sus competencias a formar profesionistas en el arte del cuidado ambiental y sustentable, sin embargo, actualmente se sugiere que todos los programas o currículos de cualquier campo, llámese sociología, economía, medicina, etc. lleve implícito el elemento medio ambiente pero con un enfoque sustentable, y transversal, es decir, que cada ciencia relacione sus objetivos con lo que acontece en el medio ambiente y su relación con el desarrollo sustentable. No es suficiente que una universidad cuente con escuelas o facultades que formen profesionistas en el campo de las ciencias ambientales.

Para concluir y a manera de sugerencia, los problemas ambientales a nivel mundial están terminando con la naturaleza, con la biodiversidad, la Educación Ambiental puede contribuir de manera satisfactoria en su preservación y cuidado por lo tanto, se sugiere revisar los beneficios de integrar la Educación Ambiental con enfoque sustentable en el Currículo.

\section{REFERENCIAS}

ACOSTA, Sylvia (2000), Una propuesta para evitar la disociación de lo natural y lo social. UAM, México, UAM.

ALCÀNTARA, M. et al., (2006), "Educación ambiental, biodiversidad, Espacios Naturales y naturaleza. III”(JORNADAS DE EDUCACIÓN AMBIENTAL DE LA COMUNIDAD AUTÓNOMA DE ARAGÓN. 24, 25 Y 26 DE MARZO DE 2006 • CIAMA, LA ALFRANCA,

ZARAGOZA),http://www.google.co.za/url?sa=t\&rct=j\&q=\&esrc=s\&source=web\&cd= 1\&ved=0CBoQFjAAahUKEwjytZ_fl4jJAhXEOBoKHTUkCp0\&url=http\%3A\%2F\%2 Fwww.aragon.es\%2Festaticos\%2Fcelia\%2FBIODIVERSIDAD.pdf\&usg=AFQjCNG_ w2VXsGDaM8MXX1_LcJfQVfoU3Q[Consulta: abr. 2016].

BEDOLLA, Ramón et al., (2014), "La ambientalización curricular de los planes de estudio en la Universidad Autónoma de Guerrero. Caso Programa de Sociología", Revista Tlamati, Núm. 3, Universidad Autónoma de Guerrero, pp. 14-21.

BEDOLLA, Ramón et al.,(2015),"Evaluación del Desempeño acorde a las Competencias Docentes del nivel superior en la UAGro. Unidad Académica de 


\section{CIÊNCIAS HUMANAS}

REVISTA DO PROGRAMA DE PÓS-GRADUAĞ̈O EM EDUCAĞ̄o

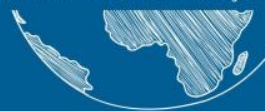

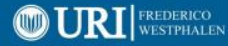

Enfermería”, Revista Foro de Estudios sobre Guerrero, Núm. 1, Consejo de Ciencia y Tecnología, pp. 1-11.

BEDOLLA, Ramón et al., (2016), "La educación ambiental para la sustentabilidad a considerar en el diseño de un currículo", Revista Atlante: Cuadernos de Educación y Desarrollo. En línea: http://www.eumed.net/rev/atlante/2016/02/curriculo.html

BRAVO, M. Teresa (2012), “La UNAM y sus procesos de ambientalización curricular. Revista Mexicana de Investigación Educativa", Núm. 55, Scielo. http://www.scielo.org.mx/scielo.php?script=sci_arttext\&pid=S140566662012000400006@ COPYRIGHT,www.portelizabethforever.co.za. , http://www.portelizabethforever.co.za/port-elizabeth-fauna.html[Consulta: abr. 2016].

CONAM, Consejo Nacional del Ambiente (S/F), "Educación Ambiental como tema transversal. Manual para trabajar en la programación de aula. Lima, Perú", (Consejo Nacional del Ambienta (CONAM). Educación Ambiental como tema transversal. Manual para trabajar en la programación de aula),Perú. pp.8-11.

CIUDADES.CO, "Localidad de Port Elizabeth" (Eastern Cape), http://www.ciudades.co/sudafrica/ciudad_port-elizabeth_6070.html[Consulta: abr. 2016]. Definición ABC, "TU DICCIONARIO HECHO FACIL". (Definición de Biodiversidad), http://www.definicionabc.com/medioambiente/biodiversidad.php[Consulta: nov. 2015].

ESPEJEL, Adelina et al., (2012), "Educación ambiental escolar y comunitaria en el nivel medio superior", Puebla-Tlaxcala. Revista Mexicana de Investigación Educativa, Núm. (55), SciELO, pp. 1-2.

FIGUEROA, Eugenio (2005), Valoración, usos y perspectivas ¿Hacia dónde va Chile?, Chile, Universitaria.https://books.google.co.za/books?id=BEFuszdUG$\mathrm{kC} \& \mathrm{pg}=\mathrm{PA} 322 \& \mathrm{dq}=$ concepto+de+biodiversidad\&hl=en\&sa=X\&ved=0CBoQ6AEwA GoVChMI9aCsSDyQIVRA4PCh1sUwnN\#v=onepage\&q=concepto\%20de\%20biodiver sidad\&f=false[Consulta: abr. 2016].

GARCÌA, Abisai et al., (2004), "Biodiversidad de Oaxaca. Oaxaca”, México: Instituto de Biología, UNAM.

https://books.google.co.za/books?id=TQfX0cL3ieQC\&pg=PA21\&dq=concepto+de+bio diversidad\&hl=en \&sa=X\&ved=0CCEQ6AEwAWoVChMI9aCs-

rSDyQIVRA4PCh1sUwnN\#v=onepage $\& \mathrm{q}=$ concepto $\% 20 \mathrm{de} \% 20$ biodiversidad $\& \mathrm{f}=$ false , [Consulta: abr. 2016].

GONZÀLEZ, M. CARMEN (2005), González; M. M. C. (1996),“Principales tendencias y modelos de la Educación ambiental en el sistema escolar", Revista Iberoamericana de Educación, Nùm.11, pp.13-74. 


\section{OCURI|}

GONÀLEZ, Edgar et al.,(2000),“La Educación Ambiental en México: Logros, perspectivas y retos de cara al nuevo milenio". México, Secretaría de Medio Ambiente, Recursos Naturales y pesca. Presentado en Congreso III Congreso Iberoamericano de Educación Ambiental. México.p.3.

LARA, Joséet al.,(2010),“Representación social de las causas de los problemas ambientales. El caso de la Benemérita Universidad Autónoma de Puebla", RevistaTrayectorias, Núm. 30, p. 40.

MACHADO, Susana (2002), "La importancia de la Educación Ambiental en la protección de la biodiversidad de Brasil”,Maestría en Educación Ambiental de la Universidad de la Florida EUA, http://www.google.co.za/url?sa=t\&rct=j\&q=\&esrc=s\&source=web\&cd=1\&ved=0CB0 QFjAAahUKEwiPhdidkojJAhXHRBQKHabzA30\&url=http\%3A\%2F\%2Fdc.itamaraty. gov.br\%2Fimagens-e-textos\%2Frevistaesp9-

mat7.pdf\&usg=AFQjCNHDWeXCB9uAAaJtvoXEm4SshfRK4g\&bvm=bv.107406026, bs.2,d.ZWU[Consulta: nov. 2015]. pp.51-52.

MARTİNEZ, Roger (2010), "La importancia de la educación ambiental ante la problemática actual”, Revista Educare, Núm. 1, p. 1.

MARTİN, B. L. et al., (2007), "Biodiversidad y bienestar humano: el papel de la diversidad funcional”, Revista Ecosistemas, Num.3 p. 1.

NACIONES UNIDAS, (2002), "Informe de la Cumbre Mundial sobre el Desarrollo Sostenible. Johannesburgo (Sudáfrica)”. Nueva York. pp.3,53.

http://www.cepal.org/rio20/noticias/paginas/6/43766/WSSD_Informe.ESP.pdf[Consulta : abr. 2016].

NELSON MANDELA METROPOLITAN UNIVERSITY (NMMU), Fortomorrow. (2016), "Undergraduate PROGRAMMES General information and Admission Requirements", (MyJourney: Port Elizabeth, South Africa) pp. 2,36-39.

NIETO, L. María (2001), “Modalidades de la Educación Ambiental: diversidad y desafíos”, Brasil, Rima.

NIETO, L. María. et al., (2008). Guía para la Estructuración y Programación de un Proyecto de Educación Ambiental y para la Sustentabilidad. México.

ORGANIZACIÓN DE LAS NACIONES UNIDAS PARA LA EDUCACIÓN Y DIVERSIFICACIÓN, LA CIENCIA Y LA CULTURA (UNESCO) ((2005-

2014), "Educación para el Desarrollo Sostenible (EDS)", Consultado en: http://www.unesco.org/new/es/our-priorities/sustainable-development/[Consulta: nov. 2015]. 


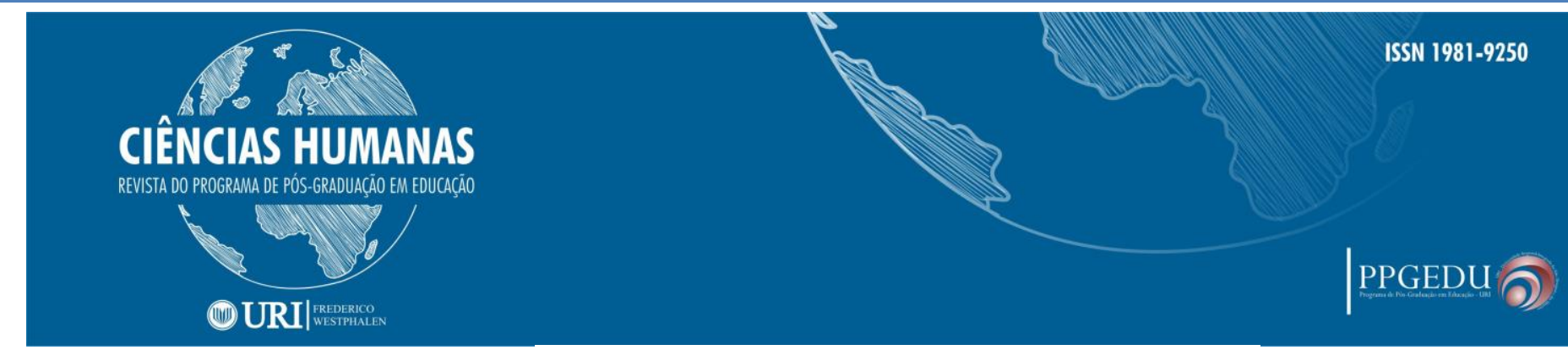

SOUTHAFRICA.INFO "Brand South Africa's information gateway to South

Africa",http://www.southafrica.info/about/geography/geography.htm\#.VwPThJx96Ukht tp://www.unesco.org/new/es/our-priorities/sustainable-development/[Consulta: nov.

2015].

South Africa Yearbook 2011/12 - Environment. Consultado en:

http://www.gcis.gov.za/sites/www.gcis.gov.za/files/docs/resourcecentre/yearbook/2011/ 14_Environment.pdf

TREAT J. (2006),“Environment and Education. Rights and Responsabilities”, South Africa, Salim Vally. University of Johannesburg, The Centre for Education Rights and Transformation (CERT). p.2. 